\title{
Erratum to: Differential susceptibility to maternal expressed emotion in children with ADHD and their siblings? Investigating plasticity genes, prosocial and antisocial behaviour
}

\author{
Jennifer S. Richards ${ }^{1,2}$ - Catharina A. Hartman ${ }^{3}$ Barbara Franke $^{4,5}$. \\ Pieter J. Hoekstra ${ }^{3}$ - Dirk J. Heslenfeld ${ }^{6} \cdot$ Jaap Oosterlaan $^{6}$. \\ Alejandro Arias Vásquez ${ }^{1,5} \cdot$ Jan K. Buitelaar ${ }^{1,2}$
}

Published online: 4 August 2015

(C) Springer-Verlag Berlin Heidelberg 2015

\section{Erratum to: Eur Child Adolesc Psychiatry (2015) 24:209-217 DOI 10.1007/s00787-014-0567-2}

Unfortunately, due to a coding difference between data imported from the UK IMAGE lab and the coding in our own lab at the Radboudumc, approximately 200 persons in NeuroIMAGE received a wrong genotype for the $H T T L P R$ variant. This mistake was noticed while merging new data. For the current paper a total of 37/366 children,

The online version of the original article can be found under doi:10.1007/s00787-014-0567-2.

Electronic supplementary material The online version of this article (doi:10.1007/s00787-015-0750-0) contains supplementary material, which is available to authorized users.

Jennifer S. Richards

Jennifer.Richards@radboudumc.nl

1 Department of Cognitive Neuroscience, Donders Institute for Brain, Cognition and Behaviour, Radboud University Medical Center, Nijmegen, The Netherlands

2 Karakter Child and Adolescent Psychiatry University Center, Nijmegen, The Netherlands

3 Department of Psychiatry, University of Groningen, University Medical Center Groningen, Groningen, The Netherlands

4 Department of Human Genetics, Donders Institute for Brain, Cognition and Behaviour, Radboud University Medical Center, Nijmegen, The Netherlands

5 Department of Psychiatry, Donders Institute for Brain, Cognition and Behaviour, Radboud University Medical Center, Nijmegen, The Netherlands

6 Department of Clinical Neuropsychology, VU University Amsterdam, Amsterdam, The Netherlands adolescents, and young adults received a wrong genotyping code. After correction and extensive checks, all analyses involving HTTLPR (including the cumulative plasticity index) were rerun. The new results can be found in Tables S1-S4, S6 and Table S7.

In Table $\mathrm{S} 1$ the correct genotype frequencies can be found for 5-HTT and the cumulative plasticity index. Table S7 shows the correct allele frequency for the 5-HTT short allele. Again, no deviations from Hardy Weinberg Equilibrium were found, although the $p$ value was now $p=.13$ instead of $p=.78$ (page 212; the fifth sentence under "Genotyping" in the published version). Table S2 contains the corrected child and genotype correlations for 5-HTT and the cumulative plasticity index with (anti) social behaviour and maternal EE. Once more, no significant correlations were found between maternal warmth and any of the children's plasticity genes, but all $p$ values were now $p>.079$ instead of $p>.261$ (page 213 ; the first sentence of the first paragraph under "Results" in the published version). In addition, a significant small correlation was now found between the cumulative plasticity index and maternal warmth $(r=.14, p<.01)$. Therefore, the following text should be inserted after the second sentence of the first paragraph under "Results" on page 213: "Likewise, maternal warmth was weakly correlated with the cumulative plasticity index $(r=.14, p<.01)$ ".

The correct results for 5-HTT and the cumulative plasticity index with regards to main and $\mathrm{G} \times \mathrm{E}$ effects can be found in Tables S3, S4, and S6. All results remained unchanged, with the exception of supplementary analyses in which we previously reported that two $\mathrm{G} \times \mathrm{E}$ had been found for PMEE $\times 5$-HTT, and PMEE $\times$ Cumulative plasticity index on parent reported prosocial behaviour which did not survive the correction for multiple testing. After careful reanalyses with the correct data, both reported $\mathrm{G} \times \mathrm{E}$ effects were not (nominally) significant $(p=.719$, 
$p=.144$ respectively, see Table S6). These new results lead to the redundancy of the text on page 213 of the published version and page 8 of the electronic supplementary material. Specifically, on page 213 of the published version, the last two sentences under the sub heading "Supplementary analyses", "For DAT1 and DRD4 genotype, no main or $\mathrm{G} \times \mathrm{E}$ effects were found. However, interaction effects were found for PMEE $\times 5$-HTT, and PMEE $\times$ Cumulative plasticity index on parent-reported prosocial behaviour. These findings did not survive correction for multiple testing and were opposite to the direction previously reported in the literature" should read as "No main or G $\times$ E effects were found".

In the electronic supplementary material the last three sentences under the heading "High versus low positive maternal expressed emotion" on page 8, "In addition, interaction effects were found for PMEE $\times 5$-HTT and PMEE $\times$ Cumulative plasticity on parent reported prosocial behaviour. Contrary to the hypothesis, children with no copies of the HTTLPR short allele had more prosocial behaviour when their mothers showed high PMEE, than when mothers expressed low PMEE. The same pattern was found for children with the lowest score on the cumulative plasticity index (thus having no copies of the $D R D 4$ 7-repeat, DAT1 9-repeat or 5-HTT S allele). However, both interaction effects did not survive correction for multiple testing" was adapted to "However, here too, no significant interaction effects were found".

Finally, in the "Discussion" section of the published version, the last two sentences of the second paragraph on pages 213-214, "Only when including a categorical rather than a continuous measure of EE (high versus low PMEE) in supplementary analyses were two $\mathrm{G} \times \mathrm{E}$ interactions found; these however, were opposite to the hypothesized direction and did not survive correction for multiple testing. Finally, analyses in subsamples (e.g. boys with ADHD), which had yielded meaningful $\mathrm{G} \times \mathrm{E}$ interactions in a previous study [17], did not change the results." should be adapted to "Analyses with a categorical measure rather than a continuous measure of EE (high versus low PMEE) and in subsamples (e.g. boys with ADHD), which had yielded meaningful $\mathrm{G} \times \mathrm{E}$ interactions in a previous study [17], did not change the results." Importantly, the conclusions of this paper remain the same. 